\title{
Accounting for differences in the bioactivity and bioavailability of vitamers
}

Jesse F. Gregory III*

Food Science and Human Nutrition Department, University of Florida, Gainesville, Florida, USA

Abstract

Essentially all vitamins exist with multiple nutritionally active chemical species often called vitamers. Our quantitative understanding of the bioactivity and bioavailability of the various members of each vitamin family has increased markedly, but many issues remain to be resolved concerning the reporting and use of analytical data. Modern methods of vitamin analysis rely heavily on chromatographic techniques that generally allow the measurement of the individual chemical forms of vitamins. Typical applications of food analysis include the evaluation of shelf life and storage stability, monitoring of nutrient retention during food processing, developing food composition databases and data needed for food labeling, assessing dietary adequacy and evaluating epidemiological relationships between diet and disease. Although the usage of analytical data varies depending on the situation, important issues regarding how best to present and interpret the data in light of the presence of multiple vitamers are common to all aspects of food analysis. In this review, we will evaluate the existence of vitamers that exhibit differences in bioactivity or bioavailability, consider when there is a need to address differences in bioactivity or bioavailability of vitamers, and then consider alternative approaches and possible ways to improve the reporting of data. Major examples are taken from literature and experience with vitamin $\mathrm{B}_{6}$ and folate.

Keywords: Vitamin; vitamers; bioactivity; bioavailability; analysis; databases

$\mathrm{M}$ ost of the vitamins exist as groups of chemically related compounds having similar biological activity capable of meeting a nutritional requirement (frequently called 'vitamers'). The usage of the term vitamer dates back to the 1940s (e.g. 1). Criticisms of this terminology have been raised since the era of its inception (e.g. 2); however, this terminology will be continued for this review in view of its widespread usage and the lack of suitable alternatives.

The existence of multiple vitamers complicates many aspects of the nutrient analysis in foods and diet samples. For example, analytical method development must account for all nutritionally active forms of a vitamin. Although this would seem to be an obvious requirement, it often complicates the development, selection and interpretation of methods for food analysis in situations involving vitamers for which stability is poor or reliable reference standards cannot be obtained commercially. This is especially the case for vitamins in which substantial differences in bioactivity or bioavailability may exist. This diversity of vitamer forms also complicates the reporting and interpretation of analytical results and also complicates the development of reliable analytical methods. When methods of analysis are chosen carefully and validated properly, such procedures can provide highly reliable information regarding the vitamer forms in individual foods and diets. This ability to measure individual vitamer forms in foods constitutes a major attribute of chromatographic methods over traditional, less-specific methods.

But in spite of this modern analytical power, the following questions illustrate the complexity of this area, including: (a) In which cases of food analysis is there a need to consider differences in vitamer bioactivity and/or bioavailability? (b) Does the analytical method being used allow measurement of all biologically active vitamers? (c) Is our understanding of the bioactivity and bioavailability of particular vitamers sufficient to allow reliable adjustments of food composition data to reflect nutritional properties? (d) Can nutritional requirements and food labeling be expressed in a way that incorporates the differences in bioavailability or bioactivities among vitamers?

Whereas the existence of differences in bioactivity/ bioavailability among vitamers is widely recognized, situations differ regarding how to address this issue when interpreting and reporting analytical data. Which approach to use depends largely on the reason for the 
analysis, the particular vitamin, and the intended application of the data. In this short discussion, we will consider which of the vitamins exhibit substantial differences in bioavailability or bioactivity among vitamers, issues in the selection and/or development of assay methods, and approaches in the application of the data to make nutritional inferences.

\section{Vitamer forms, bioavailability, and bioactivity}

Before discussing how to address issues of bioavailability and bioactivity in the reporting of analytical data, a summary of the vitamins for which multiple vitamers exist is necessary as a starting point. Table 1 provides an overview of the major forms of vitamins and brief commentary regarding their dietary roles and issues pertaining to bioavailability or bioactivity. Whereas a comprehensive evaluation of this topic is beyond the scope of the present review, we will consider general approaches and identify major issues. We also will discuss the feasibility of developing generalized approaches to account for differences in bioavailability or bioactivity. The first requirement in making such assessment is defining terms and evaluating the situation with respect to the various vitamins. The reader will note that, in several cases, differentiating the terms bioavailability and bioactivity is difficult due to the overlap of these concepts and methods used for their experimental evaluation.

\section{Bioactivity}

Bioactivity refers to the biological activity (i.e. vitamin activity) of a vitamer tested relative to the appropriate reference form of that vitamin. Although this is conceptually simple, the actual ranking in bioactivity for vitamers often varies considerably in research studies and reported in the scientific literature. Such variation in apparent bioactivity of vitamers can be due to differences in response of the experimental model used (e.g. bacteria, rats, chicks, or humans), the experimental design involving acute (bolus) or chronic administration, the dose employed, and the criterion of response (e.g. animal growth or prevention of deficiency symptoms or use of a metabolic biomarker in animal or human studies).

The best characterized examples of differences in bioactivity among vitamers exist for the fat soluble vitamins. This has long been recognized and the largely discontinued system of international units was devised as a means of addressing these differences. For example, differences among the vitamin $\mathrm{E}$ vitamers have been reported on the basis of bioassays largely reflecting antioxidant activity (e.g. 3). Since bioactivity of vitamin E vitamers varies, expressing analytical data as total vitamin E yields an ambiguous result and presenting data individually for each of the tocopherols is preferable. More recent understanding of the specific biological role of $\alpha$-tocopherol has led to the development of Recommended Dietary Allowances (RDA values) for vitamin E solely on the basis of $\alpha$-tocopherol (4). For this reason, presenting data for $\alpha$-tocopherol alone also is essential in conveying vitamin E activity. The natural stereochemical form of $\alpha$-tocopherol is RRR- $\alpha$-tocopherol, which exhibits greatest vitamin $\mathrm{E}$ activity of the eight possible diastereoisomeric forms (5). Such differences among stereochemical forms must be recognized when presenting analytical data for supplements or fortified food in which other less active $\alpha$-tocopherol diastereoisomeric species may be present. For example, the fully racemic form (all-rac- $\alpha$-tocopherol) exhibits only 50\% activity of naturally occurring RRR- $\alpha$-tocopherol.

In the case of vitamin $\mathrm{K}$, analytical data are most often presented as phylloquinone, the primary form of vitamin $\mathrm{K}$ in foods. However, for foods containing lipids prepared from hydrogenated plant oils, a separate listing for dihydrophylloquinone (6) would be preferable in view of its lower vitamin $\mathrm{K}$ activity. Menaquinones constituting the vitamin $\mathrm{K}_{2}$ group are products of bacterial synthesis with variable isoprenoid chain length (7). The long chain menaquinones found commonly in human tissues presumably are derived from intestinal bacterial synthesis as well as from certain fermented foods. In contrast, the presence of menaquinone-4 (i.e. having 4 isoprenoid units) in tissues is a result of mammalian metabolic conversion from phylloquinone. The relative qualitative and quantitative activities of dietary forms of phylloquinone and menaquinone forms have not been fully determined. Evidence from epidemiological (8) and clinical studies (9) suggest that differences exist among vitamin $\mathrm{K}$ species with respect to maintaining cardiovascular and bone health. Thus, providing analytical data on the individual forms of vitamin $\mathrm{K}$ in food analysis and dietary studies is warranted.

Analytical data for vitamin $\mathrm{D}_{2}$ and $\mathrm{D}_{3}$ are generally summed, for example in the USDA National Nutrient Database for Standard Reference (10). Evidence of greater activity of vitamin $\mathrm{D}_{3}$ than $\mathrm{D}_{2}$ (11), including an activity ratio estimated to be $9.5: 1(12)$, suggests that individual listings for these vitamin $\mathrm{D}$ vitamers would be preferable to facilitate nutritional interpretation as needed. For vitamin A, similar vitamin activity is generally observed among common dietary vitamers retinol, retinaldehyde, and added retinyl esters; however, the potential exists for the generation of corresponding cis-isomers having lesser vitamin A activity as a result of the thermal processing of foods. Although the activity of cis-isomers has been determined (13), analytical data for cis-isomers of vitamin A compounds are generally not reported in food data base tabulations in view of difficulties in their measurement and variability in their extent of formation. 
Table 1. Summary of major vitamer forms of each vitamin class, with brief commentary regarding bioactivity and bioavailability considerations

\begin{tabular}{|c|c|c|}
\hline Vitamin & Chemical forms & Comments \\
\hline \multirow[t]{6}{*}{ Vitamin A } & Retinol & Approximately full bioavailability \\
\hline & Retinyl esters & Full bioavailability of retinyl acetate and palmitate \\
\hline & Retinaldehyde & Approximately equivalent to retinol \\
\hline & $\beta$-Carotene & \\
\hline & $\alpha$-Carotene & \\
\hline & $\beta$-Cryptoxanthin & \\
\hline \multirow[t]{4}{*}{ Vitamin D } & $\mathrm{D}_{3}$, Cholecalciferol & Evidence of greater activity of $D_{3}$ than $D_{2}$ \\
\hline & $\mathrm{D}_{2}$, Ergocalciferol & \\
\hline & 25-Hydroxyvitamin D forms & Lower content but higher bioavailability than parent (nonhydroxylated) vitamin \\
\hline & & D compounds \\
\hline \multirow[t]{6}{*}{ Vitamin E } & $\alpha$-Tocopherol & $\begin{array}{l}\alpha \text {-Tocopherol exhibits primary in vivo activity. Greatest activity in natural RRR } \\
\text { stereochemical form }\end{array}$ \\
\hline & $\alpha$-Tocopheryl acetate & Acetate and other esters are fully available - common form used in fortification \\
\hline & $\beta$-tocopherol & Primarily contributes antioxidant activity \\
\hline & $\delta$-tocopherol & Primarily contributes antioxidant activity \\
\hline & $\gamma$-tocopherol & Primarily contributes antioxidant activity \\
\hline & Tocotrienols & Primarily contributes antioxidant activity \\
\hline \multirow[t]{5}{*}{ Vitamin $\mathrm{K}$} & Phylloquinone $\left(\mathrm{K}_{\mathrm{I}}\right)$ & Synthesized in plants - primary dietary form \\
\hline & Menaquinone-n $\left(K_{2}, M K-n\right)$ & Bacterial synthesis \\
\hline & MK-4 & Synthetic; also produced in vivo from $\mathrm{K}_{2}$ sources \\
\hline & Dihydrophylloquinone & Produced during hydrogenation of plant oils; reduced activity \\
\hline & Menadiones $\left(\mathrm{K}_{3}\right)$ & Synthetic; highly available \\
\hline \multirow[t]{2}{*}{ Thiamin } & Thiamin & Vitamers have equivalent activity and bioavailability \\
\hline & Thiamin phosphates & \\
\hline \multirow[t]{5}{*}{ Riboflavin } & Riboflavin & Riboflavin, FAD and FMN have approximately equivalent activity and \\
\hline & & bioavailability \\
\hline & Flavin mononucleotide (FMN) & \\
\hline & Flavin adenine dinucleotide (FAD) & \\
\hline & Minor forms & Probably contribute to activity. \\
\hline \multirow[t]{6}{*}{ Niacin } & Nicotinic acid & Full niacin activity \\
\hline & Nicotinamide & Full niacin activity \\
\hline & NAD \& NADP & Highly available \\
\hline & NADH \& NADPH & Apparent gastric instability \\
\hline & Nicotinamide riboside & Probably contributes to total niacin activity in milk \\
\hline & Bound forms of niacin & Little availability unless released by alkaline treatment \\
\hline \multirow[t]{8}{*}{ Vitamin $B_{6}$} & Pyridoxine & Full activity and bioavailability \\
\hline & Pyridoxal \& pyridoxamine & Approximately equivalent to pyridoxine; occasionally reported to have slightly \\
\hline & & lower bioactivity \\
\hline & $B_{6}$ 5'-phosphate vitamers & Similar to bioavailability to nonphosphorylated vitamers \\
\hline & Pyridoxine-5'- $\beta-D$-glucoside & Approximately $50 \%$ (human) bioavailability \\
\hline & 4-Pyridoxic acid & Catabolic product inactive \\
\hline & $\varepsilon$-Pyridoxyllysine & A protein bound complex of vitamin $B_{6}$ formed during food processing/storage. \\
\hline & & $\begin{array}{l}\text { Partial ( } \sim 50 \% \text { ) bioavailability; } \varepsilon \text {-pyridoxyllysine is not detected in routine } \\
\text { methods of vitamin } B_{6} \text { analysis }\end{array}$ \\
\hline
\end{tabular}




\begin{tabular}{|c|c|c|}
\hline Vitamin & Chemical forms & Comments \\
\hline \multirow[t]{4}{*}{ Pantothenic acid } & Pantothenic acid & Full activity and bioavailability \\
\hline & Coenzyme A & Approximately full bioavailability \\
\hline & Pantothenol & Approximately full activity and bioavailability \\
\hline & 4'-Phosphopantetheine & Approximately full activity and bioavailability \\
\hline \multirow[t]{3}{*}{ Biotin } & Biotin & Common natural form: highly available \\
\hline & Biocytin ( $\varepsilon$-biotinyl lysine) & $\begin{array}{l}\text { Protein form, also derived from turnover of biotin enzymes; slower absorption } \\
\text { than free biotin }\end{array}$ \\
\hline & Catabolic products inactive & \\
\hline \multirow[t]{11}{*}{ Folate } & Folic acid & $\begin{array}{l}\text { Highly available in foods and supplements. High doses may exceed metabolic } \\
\text { capacity. }\end{array}$ \\
\hline & Naturally occurring folates: & Often incomplete bioavailability probably due to food matrix and entrapment \\
\hline & Dihydrofolate & Unstable - minor food folate \\
\hline & Tetrahydrofolate (THF) & Common natural folate: unstable; may undergo degradation if Gl tract \\
\hline & 5-Methyltetrahydrofolate & Major naturally occurring folate vitamer \\
\hline & 5-Formyltetrahydrofolate & Common natural folate \\
\hline & I0-Formyltetrahydrofolate & Common natural folate \\
\hline & 5, 10-Methenyltetrahydrofolate & Common natural folate; also formed in acidic equilibrium with 10 -formylTHF \\
\hline & 5,10-Methylenetetrahydrofolate & Readily dissociates when heated to yield THF \\
\hline & 10-Formyldihydrofolate & Oxidation product of 10 -formylTHF \\
\hline & I0-Formyl-folic acid & Oxidation product of I0-formyldihydrofolate \\
\hline \multirow[t]{4}{*}{ Vitamin $\mathrm{B}_{12}$} & Cyanocobalamin & Predominant synthetic $B_{12}$ vitamer \\
\hline & Methylcobalamin & Common vitamin $B_{12}$ coenzyme form \\
\hline & Adenosylcobalamin & Common vitamin $B_{12}$ coenzyme form \\
\hline & Aquacobalamin & Common in vivo form \\
\hline
\end{tabular}

For the case of water-soluble vitamins, the primary vitamers often exhibit only small differences in inherent bioactivity, although variability in bioavailability may occur. Approximately equivalent bioactivity appears to be the case for the principal vitamers of thiamin, niacin, riboflavin, pantothenic acid, folate, and vitamin $B_{12}$. This also is the case for vitamin $\mathrm{B}_{6}$, in spite of reports of somewhat lower activity of pyridoxal and pyridoxamine than pyridoxine in some experimental approaches (e.g. 14). Such small differences appear to have little nutritional importance. In contrast, niacin constitutes an interesting case in which, in principle, all common dietary forms (i.e. nicotinic acid, nicotinamide, NAD and NADP) are expected to have similar nutritional activity (15) but the reduced nucleotide forms NADH and NADPH appear to exhibit lower bioactivity. The observed lower bioactivity of NADH and NADPH presumably is attributable to gastric instability due to the acid lability of these vitamers (16). Changes in the proportions of niacin vitamers during corn maturation are associated with altered nutritional properties for this reason $(17,18)$. It should be noted that a form of niacin identified in milk (nicotinamide riboside) appears to contribute niacin activity but its quantitative importance in milk (19) and potentially other foods remains unclear. Although it is generally prudent to report individual vitamers in food analysis, at present there is little reason to consider adjusting results for the purpose of equating the bioactivity of vitamers at this time.

\section{Bioavailability}

The term bioavailability in the nutritional context typically is defined more broadly than in the pharmacological context. A common definition is the 'fraction of an ingested nutrient that is available for utilization in normal physiologic functions and for storage' (20). Whereas this is not universally applicable (for example, water-soluble vitamins generally do not undergo storage), the concept provides a workable framework in terms of the relative absorption and utilization of dietary vitamins. It should be recognized, however, that bioavailability is a complex and variable concept (21) that often cannot be reliably employed in databases, labeling and many forms of dietary assessment. Moreover, nutrient bioavailability in 
the context of whole meals or whole diets, as opposed to individual foods, probably is a more important consideration with respect to predicting health outcomes (22).

Mechanisms responsible for differences in bioavailability of various types of vitamins or among vitamers often can be explained on the basis of differences in intestinal absorption (including variation in stability in the GI tract as described above) and/or differences in the rate or extent of release of the active vitamer moiety from a conjugated form (e.g. pyridoxine- $\beta$-D-glucoside and various folates; see below). The vitamin A-active carotenoids exhibit variability in Retinol Activity Equivalents (RAE) and, thus, the ratios used for the calculation of RAE. These differences among carotenoids apparently can be attributed to incomplete cleavage during digestion to release retinaldehyde and also variation whether one or two components of the carotenoid molecule can yield an active form of vitamin A following carotenoid cleavage. With respect to the carotenoid example, the US Institute of Medicine has established the following conversion factors (4). The IOM (4) defined the following values in which $1 \mathrm{mg}$ retinol is assumed to be derived from: $2 \mathrm{mg}$ of supplemental $\beta$-carotene, $12 \mathrm{mg}$ dietary $\beta$-carotene, $24 \mathrm{mg} \alpha$-carotene, and $24 \mathrm{mg} \alpha$-cryptoxanthin. In spite of some disagreement regarding the merits of the IOM recommendations by certain investigators in this field (e.g. 23), this approach provides a reasonable framework for estimating the contributions of carotenoids to dietary vitamin A activity. As is the case with preformed vitamin A species, conversion of carotenoids to cis-isomers leads to reduced activity as vitamin A (24). These differences presumably are attributable to the lesser vitamin A activity of cis-forms of retinaldehyde released from the ciscarotenoid isomers.

Other examples of incomplete bioavailability and variation among vitamers exist for the $\mathrm{B}$ vitamins. In the case of 'bound' forms of niacin in grains, which are linked either to carbohydrate or as peptidyl forms $(25,26)$, very low bioavailability is observed unless the grains are subjected to alkaline treatment (e.g. as in tortilla production). With respect to vitamin $\mathrm{B}_{6}$, pyridoxine- $\beta$-D-glucoside exists commonly throughout the plant kingdom (26, 27), constitutes an important factor that affects the overall bioavailability of this vitamin in a wide range of plantderived foods. In spite of initial research showing approximately $25 \%$ bioavailability of PN-glucoside as a source of vitamin $\mathrm{B}_{6}$ in rats (28), later work showed conclusively that humans exhibited approximately $50 \%$ bioavailability for PN-glucoside (29-31). Free pyridoxine $\mathrm{HCl}$ added in food fortification undergoes nearly complete absorption.

In the case of vitamin $B_{12}$, absorption depends on proper functioning of integrated steps in digestion including the release of vitamin $B_{12}$ from dietary protein, specific binding in the stomach to haptocorrin, transfer in the small intestine to the stomach-derived $\mathrm{B}_{12}$ binding protein called intrinsic factor, and then carrier mediated absorption in the ileum. Any digestive disorder that interferes with this process, including physiological or pharmacological interference with gastric secretions, can directly interfere with vitamin $\mathrm{B}_{12}$ absorption (32). In addition, impaired gastric secretion often is associated with malabsorption of naturally occurring vitamin $\mathrm{B}_{12}$ in food even though the absorption of synthetic vitamin $\mathrm{B}_{12}$ might be unimpaired (33). Classic research by Dolscherholmen and associates (34-36) involving intrinsic enrichment of fish, eggs and chicken meat showed markedly depressed absorption of the radiolabeled vitamin $\mathrm{B}_{12}$ in patients with pernicious anemia due to impaired production of intrinsic factor (35). Such studies have been extended using accelerator mass spectrometry techniques (37).

The bioavailability of folate is a complex topic with variability reported for the bioavailability of naturally occurring folate that may be due to entrapment in the food matrix, complicated by the existence of a variety of natural tetrahydrofolates (THF) varying in polyglutamyl chain length and folic acid that may be added in food fortification. The dietary and physiological factors affecting folate bioavailability have been discussed previously $(22,38)$. Most evidence suggests that the bioavailability of naturally occurring food folates is less than that of added folic acid $(22,38)$, although caution should be exercised in interpreting literature based on animal bioassays of folate bioavailability. Whereas bioassays using rodents are useful in some applications, they may have little value in predicting the bioavailability of dietary polyglutamyl folates in human diets in view of the differences in intestinal deglutamylation mechanism and enzymes involved. Assumed incomplete deconjugation of polyglutamyl folates has led to the assumption that polyglutamylation of natural folates constitutes a mechanism responsible for incomplete bioavailability of dietary folates; however, it must be recognized that this assumption is not fully supported by the literature $(22,38)$.

Indeed, reports of relationships between folylpolyglutamate chain length and their in vivo bioavailability range from $50 \%$ to $100 \%$. These inconsistent experimental findings likely can be attributed to variation in the protocols used, dosages employed and, potentially, analytical inaccuracies. Entrapment in the food matrix (i.e. cellular structure, etc.) presumably contributes to incomplete bioavailability of food folates. Small doses of folic acid and reduced folates exhibit effective and equivalent absorption (39), whereas larger doses ( several hundred $\mu \mathrm{g})$ show differences in post-absorptive distribution and retention in vivo $(40,41)$. For this reason, one group of investigators has criticized the use of folic acid as a reference material in earlier folate bioavailability protocols (e.g. 42).

However, this reviewer considers such arguments to have little practical relevance because the primary 
objective of most such studies is to determine relative rather than absolute bioavailability, and using the form of this vitamin (i.e. folic acid) that is the common supplemental form and food fortificant as a reference makes conceptual sense as well. Studies of the bioavailability of folic acid from fortified cereal grain food products showed effective absorption (43, 44). As will be discussed further, the development of the term Dietary Folate Equivalents (DFE) constitutes an approach to account for the generally greater absorption of added folic acid than naturally occurring food folate (45).

\section{Evaluation, selection, and development of analytical methods}

\section{Principles of vitamin assay in food analysis to allow inferences}

Several principles can be stated regarding for the development or selection of methods to be used for the determination of vitamins in foods. These include: (a) ability to distinguish and individually quantify all nutritionally active vitamers; (b) ability to distinguish and individually quantify all significant precursors or provitamin forms that contribute to overall vitamin activity; (c) existence and accessibility of appropriate standards for all vitamer forms; and (d) existence of appropriate validation and quality control protocols. Unlike traditional methods in which the entire assay depends on the preparation of a single accurate working standard, measurement of individual vitamer forms requires the preparation of a standard for each compound measured. This complicates the analysis and puts greater emphasis on the proper routine standardization of the method than would be required for more traditional methods.

\section{Methods to determine total vitamin content}

Early approaches to the determination of vitamins largely relied on procedures using either microbial or chemical methods that one hoped provided a measurement of the total (i.e. aggregate) of the nutritionally active vitamer forms. More thorough examination of the response of such methods occasionally provided evidence of nonuniform response among prominent vitamer forms and, thus, a large potential for inaccuracy when measuring total vitamin content. Such issues have been reported for typical microbiological assays for vitamin $\mathrm{B}_{6}$ (e.g. 46) and folate (e.g. 47). Analytical bias also could arise in both microbiological and chemical assays for total content of vitamins if differences existed among vitamers with respect to their efficiency of extraction and/or stability during such preparative phases of assays. For reasons such as these and in view of the potential for varying bioactivity and bioavailability of vitamers, the need for improved methods of vitamin analysis is clear. Furthermore, such discussions point clearly to the importance of developing and using methods that allow accurate quantification of individual vitamers wherever possible. A need also frequently exists to differentiate between added and naturally occurring vitamers.

The advent of high performance liquid chromatography (HPLC) transformed almost all areas of vitamin analysis and greatly facilitated the measurement of individual vitamers. In spite of the power of HPLC and its widespread application, surprising practical and conceptual limitations remain in some of the methods appearing even recently in peer-reviewed literature. An old and frequently told story of analytical chemistry lectures about an inebriated fellow looking for his lost wallet illustrates one major point very well. When asked why he was looking for it under the lamp post, he responded 'because that is where the light is.' The key point in our context is that the analyst should make every effort to employ methods that can detect and quantify all relevant forms of target vitamin, not just those that are easily detected or for which standards can be readily obtained. In the area of vitamin analysis, some of the potentially excellent published methods should be viewed as inadequate because either the method used did not provide for detection of all nutritionally active vitamers or else the investigators apparently did not recognize the need for quantifying certain vitamers. The measurement of folate and vitamin $\mathrm{B}_{6}$ vitamers in foods constitute good examples of these issues, as discussed below.

Naturally occurring folate exists as an array of THF species including THF, 5-methyl-THF, 5-formylTHF, 10-formyl-THF, 5,10-methylene-THF, and 5,10methenyl-THF, with very small amounts of 7,8-dihydrofolate (DHF) also present. In natural products including plant and animal tissues and cereal grains, 10-formylTHF and 5,10-methenyl-THF together can constitute up to $\sim 15-35 \%$ of the total folate pool in certain materials (e.g. 48, 49). Although several approaches have allowed successful measurement of folate vitamers directly as their various polyglutamyl forms (e.g. 50-52), difficulty in standardizing such methods makes their routine use in food analysis difficult. The challenge of measuring all polyglutamyl forms of each vitamer species routinely is bypassed by enzymatic hydrolysis so that each vitamer species is measured as the monoglutamyl form, but verification of full hydrolysis should be performed for each type of sample analyzed (e.g. 53).

Accounting for all types of the formyl folates constitutes another problem that has not been fully addressed in some published methods. 5-formyl-THF, 10-formyl-THF and 5,10-methenyl-THF are inter-convertible depending on $\mathrm{pH}$ and temperature used in extraction, preliminary treatment, and HPLC separation (see 52). Although the 5-formyl-THF pool exhibits quite good stability; the measurement of 10 -formyl-THF is more problematic due to these interconversions and susceptibility to 
oxidation. The rapid conversion of 10 -formyl-THF to 5,10 -methenyl-THF in acidic conditions (54) such as those used in affinity chromatographic purification and reverse phase HPLC with an acidic mobile phase allows convenient measurement of this vitamer as 5,10-methenylTHF (e.g. 49, 55). Differentiation between 10-formylTHF and 5,10-methenyl-THF present in a food sample is difficult for this reason, but reporting their sum would pose no problem in nutritional interpretation of the data since these vitamers would exhibit comparable bioactivity and bioavailability as folate sources. Two oxidation products of 10-formyl-THF also must be considered: 10-formyl-DHF and 10-formyl-folic acid.

A number of analytical methods for measurement of food folate published over the past few years have provided for the measurement of 10-formyl-folic acid, presumably because of the stability and ease of handling of this compound as well its ease of preparation and commercial availability for use as a standard. However, the nutritionally active intermediate in the oxidation of 10 -formyl-THF to 10 -formyl-folic acid is 10-formylDHF, and very few published methods include provision for its measurement. Our analyses of natural (i.e. not added) folates in cereal grain foods (47), and plant tissues $(55,56)$ have shown variable but measureable amounts of 10 -formyl-DHF. It is unlikely that any significant amount of 10-formyl-DHF exists naturally in living plant and animal tissue, so the detection of 10-formyl-DHF and 10 -formyl-folic acid in freshly obtained samples represents small and largely unavoidable oxidation of 10-formyl-THF during the preparative and analytical procedures. Since postharvest handling, processing and distribution of foods provide ample opportunity for the formation of these nutritionally active oxidation products, HPLC methods of folate analysis should allow for their measurement in addition to the major natural and added folates. The advent of labeling and databases expressing Dietary Folate Equivalents (as discussed later), puts an added requirement on method selection since the procedure must differentiate between natural folates and added folic acid. Although several well-validated procedures exist for this application (e.g. 48, 57, 58), none of these has the Association of Official Analytical Chemists International (AOAC) 'Official Method' designation.

Vitamin $\mathrm{B}_{6}$ represents another example of a challenging aspect of food analysis because of its multiple vitamer forms including pyridoxine (PN)-glucoside in plants. Various validated methods exist for the measurement of $\mathrm{B}_{6}$ vitamers individually, including $\mathrm{PN}$-glucoside (e.g. 27, 28, 59). This approach is needed to allow the user to make nutritional inferences by accounting for the reduced bioavailability of PN-glucoside. It is disconcerting that a number of published procedures for the measurement of vitamin $B_{6}$ either neglect the glycosylated form entirely or else hydrolyze it to pyridoxine and measure total pyridoxine. The latter approach would be sufficient for animal-derived foods, but for plant foods that contain a substantial fraction of vitamin $\mathrm{B}_{6}$ as PN-glucoside, such methods would lead to overestimation of overall nutritional content since the resulting data would not reflect the content and partial bioavailability of $\mathrm{PN}$-glucoside. Whereas the absence of a commercial PN-glucoside standard complicates both the development of new methods and the calibration of existing procedures, simple and reliable methods do exist for its biosynthesis and purification (60).

\section{Considering the analytical context}

As mentioned earlier, the measurement of vitamins is conducted for many reasons and in many situations. Whether there is a need to make nutritional inferences adjusting for differences in bioactivity and/or bioavailability depends on the particular situation. The following illustrates variation in context and usage of data.

(1) Analysis for process monitoring and process control: When analyses are conducted in a food manufacturing setting to monitor nutrient stability for processing control, accuracy of vitamin addition during fortification, or to evaluate shelf life, the resulting data are largely for internal use. Thus, there is little need for the reporting of data to incorporate adjustment for the variations in bioactivity or bioavailability among vitamers.

(2) Nutritional labeling: Nutrition information panels in such labeling generally are derived from databases generated by the manufacturer from direct analysis of finished food products with representative sampling, appropriate analysis and statistical evaluation. Sound analytical data are required in developing such databases to support the content claims made on the label, although the specifics of nutritional labeling policy, including analytical requirements, vary from country to country (e.g. 61, 62). The purpose of nutritional labeling is to convey to the consumer actual nutritional content, on a per serving basis, to facilitate food selection and dietary formulations by interested consumers, rather than being a detailed data sheet or certificate of analysis for the contents of each package. As practiced in the United States, the Food and Drug Administration (FDA) policy requires the content of naturally occurring vitamins in the package to be present at no less than $80 \%$ of the amount shown on the nutrition label (62). In contrast, the content of added vitamins must be no less than $100 \%$ of the label value (62). Because this requirement applies throughout the shelf life of the product during which losses of vitamin content can occur, appropriate 
overages in the level of fortification are allowed. Both analytical and compositional variability must be taken into account. For the purpose of nutritional labeling, regulatory agencies generally expect the use of validated methods with some form of official designation such as the 'Official Methods' of the AOAC. Indeed, the FDA (62) has stated: 'FDA's continuing policy since the 1970s assigns the manufacturer the responsibility for assuring the validity of a product label's stated nutrient values. Accordingly, the source of the data used to calculate nutrition label values is the prerogative of the manufacturer, but FDA's policy recommends that the nutrient values for labeling be based on product composition, as determined by laboratory analysis of each nutrient. FDA continues to recommend the use of the Official Methods of the AOAC, with non-AOAC Official Methods used only in the absence of appropriate AOAC validated methods. For each product that is included in a nutrition labeling data base submitted to FDA, the agency requests that the developer include a table identifying proposed analytical methods that were used in the analysis of each nutrient, with accompanying information containing validation of the method used by the onsite or commercial laboratory for the matrix of interest.' Unfortunately, information regarding the actual methods used and the validation protocols employed to generate these databases are not easily determined by users. One should recognize that many modern analytical methods and approaches exist that meet current needs in vitamin analysis, particularly with respect to proper measurement of individual vitamers. Unfortunately, most of these have not been evaluated for 'Official Method' status or otherwise designated as validated methods even though scientific validation has been performed. A distinct need exists for support of methods development but a great need also exists for validation of more modern methods to achieve official methods status and regulatory approval.

(3) Food Composition Databases. The expansion of food composition data bases such as the USDA National Nutrient Database for Standard Reference (10) constitutes a major service that makes food composition data readily accessible to the scientific community and the public. These facilitate evaluation of dietary adequacy in many facets of human nutrition, including clinical nutrition. The food composition databases also serve as powerful tools in evaluating diet-disease relationships in clinical and epidemiological studies. Unlike databases used for nutritional labeling of foods, the listings in the USDA National Nutrient Database for Standard
Reference (10) provide much more complete array of data for total nutrient content and a much more comprehensive listing of major vitamer forms that allow listings and inferences regarding the primary natural and added forms of vitamins, as illustrated in Table 2. The USDA database and its online format illustrate many very positive attributes and serve as a very good example for making food composition data available to a wide range of users. In spite of the breadth and depth of coverage, several aspects could be improved greatly to facilitate interpretation by interested users. First, there should be a mechanism by which the user could determine the analytical method employed. For example, being able to determine whether a niacin assay involved acid or alkaline extraction would help a knowledgeable user to determine whether the result listed reflected total (alkaline extraction/ hydrolysis) or biologically available (acid extraction/hydrolysis). This and many similar questions could be easily resolved if reference citations or links to methodologies were provided. Second, there is currently no way to ascertain the quality of the data. Providing some type of linkage to the sampling and quality control procedures used also would aid interested users in assessing data listings. Finally, users would benefit if the procedures for adjusting for differences in bioactivity and/or bioavailability were clearly defined. For example, links to a summary with definitions of terms such as Retinol Activity Equivalents and Dietary Folate Equivalents. As currently presented, the user cannot readily interpret the meaning or intent of such terms used throughout the database.

\section{Suggestions for improving the reporting of data}

The recommendations above would benefit the USDA National Nutrient Database for Standard Reference (10) but also would be applicable to and would benefit all other food composition databases.

With respect to the vitamins used as primary examples in this review, vitamin $\mathrm{B}_{6}$ and folate, this author proposes several additional recommendations. For vitamin $\mathrm{B}_{6}$ in fruits and vegetables (which account for approximately half of dietary vitamin $\mathrm{B}_{6}$ intake), a system for expressing total available vitamin $\mathrm{B}_{6}$ adjusting for the incomplete bioavailability of PN-glucoside should be implemented. PN-glucoside exhibits approximately 50\% bioavailability in humans (29-31). For this purpose, a term such as 'Bioavailable Vitamin $\mathrm{B}_{6}$ Equivalents' would be defined as: [total vitamin $\left.\mathrm{B}_{6}\right]-(0.5 *$ [PN-glucoside] $)$. This is an oversimplification since it adjusts only for the well-characterized incomplete bioavailability of PNglucoside and ignores other factors affecting vitamin $\mathrm{B}_{6}$ bioavailability; however, this would serve as a useful 
Table 2. Vitamins listed in the USDA National Nutrient Database for Standard Reference (Adapted from (7)). The various listing for the vitamins are shown in the first column, units in the second column, while the author's comments regarding the applications and rationale of the listing and measurement

\begin{tabular}{|c|c|c|}
\hline Vitamin & Units & Comments \\
\hline \multicolumn{3}{|l|}{ Fat-soluble vitamins } \\
\hline Vitamin A, RAE & $\mu \mathrm{g}, \mathrm{RAE}$ & $\begin{array}{l}\text { Vitamin A activity adjusted for differences in bioavailability/bioactivity between } \\
\text { preformed vitamin A and vitamin A-active carotenoids }\end{array}$ \\
\hline Retinol & $\mu g$ & Individual values allow calculation of RAE \\
\hline Carotene, beta & $\mu g$ & Individual values allow calculation of RAE \\
\hline Carotene, alpha & $\mu g$ & Individual values allow calculation of RAE \\
\hline Cryptoxanthin, beta & $\mu g$ & Individual values allow calculation of RAE \\
\hline Vitamin A, IU & IU & Vitamin A activity expressed in the IU system \\
\hline Lycopene & $\mu g$ & A carotenoid of interest regarding diet and health \\
\hline Lutein + Zeaxanthin & $\mu g$ & A carotenoid of interest regarding diet and health \\
\hline Vitamin E (alpha-tocopherol) & $\mathrm{mg}$ & Considered the major vitamin E-active tocopherol \\
\hline Vitamin E, added & $\mathrm{mg}$ & Added vitamin E expressed as alpha-tocopherol \\
\hline Tocopherol, beta & $\mathrm{mg}$ & A tocopherol contributing antioxidant activity \\
\hline Tocopherol, gamma & $\mathrm{mg}$ & A tocopherol contributing antioxidant activity \\
\hline Tocopherol, delta & $\mathrm{mg}$ & A tocopherol contributing antioxidant activity \\
\hline Vitamin $D\left(D_{2}+D_{3}\right)$ & $\mu g$ & Total vitamin $D$ (assumes $=$ activity of $D_{2} \& D_{3}$ ) \\
\hline Vitamin D & IU & Vitamin D activity expressed in the IU system \\
\hline Vitamin K (phylloquinone) & $\mu g$ & Primary natural vitamin $\mathrm{K}$ vitamer \\
\hline Vitamin C (total ascorbic acid) & $\mathrm{mg}$ & Ascorbic acid plus dehydroascorbic acid \\
\hline Thiamin & $\mathrm{mg}$ & Total of thiamin and thiamin phosphates \\
\hline Riboflavin & $\mathrm{mg}$ & Total of free riboflavin, FAD and FMN \\
\hline Niacin & $\mathrm{mg}$ & Total niacin, sum of nicotinamide, nicotinic acid, NAD and NADP \\
\hline Pantothenic acid & $\mathrm{mg}$ & Total pantothenic acid in microbial assay \\
\hline Vitamin $\mathrm{B}_{6}$ & $\mathrm{mg}$ & Total of all vitamers (PN-glucoside included in pyridoxine total) \\
\hline Folate, total & $\mu g$ & Total folate, largely microbial assay \\
\hline Folic acid & $\mu g$ & $\begin{array}{l}\text { Added folic acid, assumed to have } 1.7 \text { times greater bioavailability than natural } \\
\text { dietary folate }\end{array}$ \\
\hline Folate, food & $\mu g$ & Total naturally occurring folate \\
\hline Folate, DFE & $\mu g$ DFE & $\begin{array}{l}\text { Total folate expressed as dietary folate equivalents giving greater weight to folic acid } \\
\text { assuming its greater bioavailability }\end{array}$ \\
\hline Choline, total & $\mathrm{mg}$ & Sum of free choline, phosphorylcholine and phosphatidylcholine \\
\hline Betaine & $\mathrm{mg}$ & A choline catabolite of interest as an alternative methyl donor \\
\hline Vitamin $B_{12}$ & $\mu g$ & Total vitamin $B_{12}$ \\
\hline Vitamin $B_{12}$, added & $\mu g$ & Added cyanocobalamin, assumed to have greater bioavailability (basis of RDA) \\
\hline
\end{tabular}

framework. The strengths and limitations of the 'Dietary Folate Equivalent' concept have been discussed previously. 'Dietary Folate Equivalents' are defined as $\mu \mathrm{g}$ food folate $+(1.7 \mathrm{x} \mu \mathrm{g}$ synthetic folic acid $)$, with the 1.7 multiplier based on the ratio of assumed bioavailability of folic acid added to food (assumed 85\%) divided by the assumed bioavailability of natural dietary folate (assumed $50 \%$ ) (45). Whereas the merits of the assumptions of absolute and relative bioavailabilities on which the multiplier is based can be argued, the concept and approach are useful in illustrating the greater bioavailability of the added folic acid (22). Since the Dietary Folate Equivalents are firmly entrenched in widespread usage and even serve as a basis for the folate RDA, making changes in this approach is not recommended. However, users should recognize that uncertainty does exist in the quantitative concept of Dietary Folate Equivalents.

\section{Summary, conclusions, and future directions}

In summary, this review has illustrated many applications of the measurement of vitamins and has presented many examples illustrating the need for the measurement of individual vitamers wherever possible. Since major objectives of nutritional labeling and food composition databases are to allow the user to make inferences pertaining to food selection and diet composition, the presentation of analytical data that reflects nutritional properties to the greatest extent possible should remain 
an objective. All databases that incorporate terms reflecting aggregate bioactivity of vitamer forms should clearly make the definitions clearly accessible to the users.

Furthermore, making information available about the analytical methods and quality control protocols used would greatly facilitate the interpretation of data by scientific users. Finally, despite major advances in the analytical chemistry of vitamin analysis, many needs exist. In particular, new methods should allow the measurement of individual vitamers and should avoid approaches in which groups of vitamers are converted to single analyte forms. Support from users and agencies maintaining databases also is needed for the validation and certification of new analytical methods to augment and/or replace outdated 'official methods.'

\section{Conflict of interest and funding}

The author declares no conflict of interest and has not received any funding or benefits from industry or elsewhere to conduct this study.

\section{References}

1. Burk D, Winzler RJ. Heat-labile, avidin-uncombinable, species specific and other vitamers of biotin. Science 1943; 97: 57-60.

2. William RJ. Isotel, isotelic. Science 1943; 98: 386.

3. Machlin LJ, Vitamin E. In: Machlin LJ, ed. Handbook of vitamins, second edition. revised and expanded. New York: M. Dekker; 1991. p. 99-144.

4. Institute of Medicine (IOM) (2000). Dietary reference intakes for vitamin C, vitamin E, selenium, and carotenoids. Standing Committee on the Scientific Evaluation of Dietary Reference Intakes, Food and Nutrition Board, Institute of Medicine, National Academy of Sciences. Washington, DC: National Academy Press.

5. Weiser H, Vecchi M. Stereoisomers of $\alpha$-tocopheryl acetate. II. Biopotencies of all eight stereoisomers, individually or in mixtures, as determined by rat resorption-gestation tests. Int $\mathbf{J}$ Vit Nutr Res 1982; 32: 351-70.

6. Booth SL, Lichtenstein AH, O'Brien-Morse M, McKeown NM, Wood RJ, Saltzman E, et al. Effects of a hydrogenated form of vitamin $\mathrm{K}$ on bone formation and resorption. Am J Clin Nutr Dec 2001; 74: 783-90.

7. Suttie JW, Vitamin K. In: Zempleni J, Rucker RB, McCormick DB, Suttie JW, eds. Handbook of vitamins, fourth edition. Boca Raton, FL: CRC Press; 2007. p. 111-52.

8. Beulens JW, Bots ML, Atsma F, Bartelink ML, Prokop M, Geleijnse JM, et al. High dietary menaquinone intake is associated with reduced coronary calcification. Atherosclerosis 2009; 203: 489-93.

9. Knapen MH, Schurgers LJ, Vermeer C. Vitamin $\mathrm{K}_{2}$ supplementation improves hip bone geometry and bone strength indices in postmenopausal women. Osteoporos Int 2007; 18: 963-72.

10. U. S Department of Agriculture, Agricultural Research Service, USDA Nutrient Data Laboratory (2010). USDA National Nutrient Database for Standard Reference, Release No. 23. http://www.ars.usda.gov/nutrientdata. [cited 4 April 2010].

11. Houghton L, Vieth R. The case against ergocalciferol (vitamin $\mathrm{D}_{2}$ ) as a vitamin supplement. AJCN 2006; 84: 694-7.
12. Armas LA, Hollis BW, Heaney RP. Vitamin $\mathrm{D}_{2}$ is much less effective than vitamin $\mathrm{D}_{3}$ in humans. J Clin Endocrinol Metab 2004; 89: 5387-91.

13. Ames SR. Bioassay of vitamin A compounds. Fed Proc. Fed Am Soc Exp Biol 1965; 24: 917-23.

14. Wozenski JR, Leklem JE, Miller LT. The metabolism of small doses of vitamin B-6 in men. J Nutr 1980; 110: 275-85.

15. Henderson LM. Niacin Annu Rev Nutr 1983; 3: 289-307.

16. Carpenter KJ, Schelstraete M, Vilicich VC, Wall JS. Immature corn as a source of niacin for rats. J Nutr 1988; 118: 165-9.

17. Wall JS, Young MR, Carpenter KS. Transformation of niacincontaining compounds in corn during grain development. Relationship to niacin nutritional availability. J Agric Food Chem 1987; 35: 752-8.

18. Wall JS, Carpenter KJ. Variation in availability of niacin in grain products. Changes in chemical composition during grain development and processing affect the nutritional availability of niacin. Food Technol 1988; 42: 198-204.

19. Bogan KL, Brenner C. Nicotinic acid, nicotinamide, and nicotinamide riboside: a molecular evaluation of NAD+ precursor vitamins in human nutrition. Annu Rev Nutr 2008; 28: 115-30. Review.

20. Jackson MJ. The assessment of bioavailability of micronutrients: introduction. Eur J Clin Nutr 1997; 1 (51 Suppl): S1-2.

21. Bronner F. Nutrient bioavailability, with special reference to calcium. J Nutr 1993; 123: 797-802. Review.

22. Gregory JF, Quinlivan EP, Davis SR. Integrating the issues of folate bioavailability, intake and metabolism in the era of fortification. Trends in Food Sci \& Technol 2005; 16: 229-40.

23. West CE, Eilander A, van Lieshout M. Consequences of revised estimates of carotenoid bioefficacy for dietary control of vitamin A deficiency in developing countries. J Nutr 2002; 132 (9 Suppl): S2920-6.

24. Zechmeister L. Stereoisomeric provitamins A. Vitam Hormones 1949; 7: 57-81.

25. Carter EG, Carpenter KJ. The bioavailability for humans of bound niacin from wheat bran. Am J Clin Nutr 1982; 36: 855-61.

26. Gregory JF. Nutritional properties and significance of vitamin glycosides. In: McCormick DB, ed. Annual review of nutrition. Palo Alto, CA: Annual Reviews; 1998. p. 277-96.

27. Gregory JF, Ink SL. Identification and quantification of pyridoxine $\beta$-glucoside as a major form of vitamin $\mathrm{B}_{6}$ in plant-derived foods. J Agric Food Chem 1987; 35: 76-82.

28. Ink SL, Gregory JF, Sartain DB. The determination of pyridoxine- B-glucoside bioavailability in the rat. J Agric Food Chem 1986; 34: 857-62.

29. Nakano H, McMahon LG, Gregory JF. Pyridoxine-5'-betaglucoside exhibits incomplete bioavailability as a source of vitamin B-6 and partially inhibits the utilization of co-ingested pyridoxine in humans. J Nutr 1997; 127(8): 1508-13.

30. Gregory, JF. Bioavailability of vitamin $B_{6}$. Eur J Clin Nutr 1997; 51(suppl.): S43-8.

31. Gregory JF, Trumbo PR, Bailey LB, Toth JP, Baumgartner TG, Cerda JJ. Bioavailability of pyridoxine-5'-beta-D-glucoside determined in humans by stable-isotopic methods. J Nutr 1991; 121: 177-86.

32. Corcino JJ, Waxman S, Herbert V. Absorption and malabsorption of vitamin $B_{12}$. Am J Med 1970; 48: 562-9. Review.

33. Carmel R, Sinow RM, Siegel ME, Samloff IM. Food cobalamin malabsorption occurs frequently in patients with unexplained low serum cobalamin levels. Arch Intern Med 1988; 148: 1715-9.

34. Doscherholmen A, McMahon J, Ripley D. Vitamin B 12 absorption from eggs. Proc Soc Exp Biol Med 1975; 149: 987-90. 
35. Doscherholmen A, McMahon J, Ripley D. Vitamin $B_{12}$ assimilation from chicken meat. Am J Clin Nutr 1978; 31: 825-30.

36. Doscherholmen A, McMahon J, Economon P. Vitamin $B_{12}$ absorption from fish. Proc Soc Exp Biol Med 1981; 167: 480-4.

37. Carkeet C, Dueker SR, Lango J, Buchholz BA, Miller JW, Green $\mathrm{R}$, et al. Specific ${ }^{14} \mathrm{C}$-labeling of cobalamin and accelerator mass spectrometry underlie a quantitative test for $\mathrm{B}_{12}$ absorption in humans. Proc Nat Acad Sci USA 2006; 103: 5694-9.

38. Gregory JF. Bioavailability of folate. Eur J Clin Nut 1997; 51(suppl): S54-9.

39. Bhandari SD, Gregory JF. Folic acid, 5-methyl-tetrahydrofolate and 5-formyl-tetrahydrofolate exhibit equivalent intestinal absorption, metabolism and in vivo kinetics in rats. J Nutr 1992; 122: 1847-54.

40. Gregory JF, Bhandari SD, Bailey LB, Toth JP, Baumgartner TG, Cerda JJ. Relative bioavailability of deuterium-labeled monoglutamyl tetrahydrofolates and folic acid in human subjects. Am J Clin Nutr 1992; 55: 1147-53.

41. Wright AJA, Finglas PM, Dainty JR, Hart DJ, Wolfe CA, Southon $\mathrm{S}$, et al. Single oral doses of ${ }^{13} \mathrm{C}$ forms of pteroylmonoglutamic acid and 5-formyltetrahydrofolic acid elicit differences in short term kinetics of labelled and unlabeled folates in plasma: potential problems in interpretation of folate bioavailability studies. Br J Nutr 2003; 90: 363-71.

42. Wright AJ, King MJ, Wolfe CA, Powers HJ, Finglas PM. Comparison of (6 S)-5-methyltetrahydrofolic acid vs. folic acid as the reference folate in longer-term human dietary intervention studies assessing the relative bioavailability of natural food folates: comparative changes in folate status following a 16-week placebo-controlled study in healthy adults. Br J Nutr 2010; 103: 724-9.

43. Pfeiffer CM, Rogers LM, Bailey LB, Gregory JF. Absorption of folate from fortified cereal-grain products and of supplemental folate consumed with or without food determined by using a dual-label stable-isotope protocol. Am J Clin Nutr 1997; 66(6): 1388-97.

44. Johansson M, Witthoft CM, Bruce A, Jagerstad M. Study of wheat breakfast rolls fortified with folic acid. Eur J Nutr 2002; 41: $279-86$.

45. Institute of Medicine (IOM) (1998). Dietary reference intakes: thiamin, riboflavin, niacin, vitamin $\mathrm{B}_{6}$, folate, vitamin $\mathrm{B}_{12}$, pantothenic acid, biotin, and choline. Standing Committee on the Scientific Evaluation of Dietary Reference Intakes, Food and Nutrition Board, Institute of Medicine, National Academy of Sciences. Washington, DC: National Academy Press.

46. Gregory JF. Relative activity of the nonphosphorylated B-6 vitamers for Saccharomyces uvarum and Kloeckera brevis in vitamin B-6 microbiological assay. J Nutr 1982; 112: 1643-7.

47. Phillips DR, Wright AJ. Studies on the response of Lactobacillus casei to different folate monoglutamates. Br J Nutr 1982; 47(2): 183-9.

48. Pfeiffer CM, Rogers LM, Gregory JF. Determination of folate in cereal-grain food products using tri-enzyme extraction and combined affinity and reverse-phase liquid chromatography. J Agric Food Chem 1997; 45: 407-13.

49. Díaz de la Garza R, Quinlivan EP, Klaus S, Basset GJC, Gregory JF, Hanson AD. Folate biofortification in tomatoes by engineering the pteridine branch of folate synthesis. Proc Natl Acad Sci USA 2004; 101: 13720-5.

50. Seyoum E, Selhub J. Combined affinity and ion pair column chromatographies for the analysis of food folate. J Nutr Biochem 1993; 4: 488-93.
51. Bagley PJ, Selhub J. Analysis of folate form distribution by affinity followed by reversed- phase chromatography with electrical detection. Clin Chem 2000; 46: 404-11.

52. Garratt LC, Ortori CA, Tucker GA, Sablitzky F, Bennett MJ, Barrett DA. Comprehensive metabolic profiling of mono- and polyglutamated folates and their precursors in plant and animal tissue using liquid chromatography/negative ion electrospray ionisation tandem mass spectrometry. Rapid Commun Mass Spectrom 2005; 19: 2390-8

53. Engelhardt R, Gregory J. Adequacy of enzymatic deconjugation in quantification of folate in foods. J Agric Food Chem 1990; 38: 154-8.

54. Robinson DR. The nonenzymatic hydrolysis of N5,N10-methenyltetrahydrofolic acid and related reactions. In: Chytl F, ed., Methods in enzymology. San Diego, CA: Academic Press; 1971; Vol. 18B, pp. 716-25.

55. Goyer A, Collakova E, Díaz de la Garza R, Quinlivan EP, Williamson J, Gregory JF, et al. 5-Formyltetrahydrofolate is an inhibitory but well tolerated metabolite in Arabidopsis leaves. J Biol Chem 2005; 280: 26137-42.

56. Orsomando G, de la Garza RD, Green BJ, Peng M, Rea PA, Ryan TJ, et al. Plant gamma-glutamyl hydrolases and folate polyglutamates: characterization, compartmentation, and cooccurrence in vacuoles. J Biol Chem 2005; 280: 28877-84.

57. Konings EJ. A validated liquid chromatographic method for determining folates in vegetables, milk powder, liver, and flour. J AOAC Int 1999; 82: 119-27.

58. Póo-Prieto R, Haytowitz DB, Holden JM, Rogers G, Choumenkovitch SF, Jacques PF, et al. Use of the affinity/ HPLC method for quantitative estimation of folic acid in enriched cereal-grain products. J Nutr 2006; 136: 3079-83.

59. Gregory JF, Sartain DB. Improved chromatographic determination of free and glycosylated forms of vitamin $\mathrm{B}_{6}$ in foods. J Agric Food Chem 1991; 39: 899-905.

60. Gregory JF, Nakano H. Preparation of nonlabeled, tritiated, and deuterated pyridoxine-5'- $\beta$-D-glucoside and assay of pyridoxine-5'- $\beta$-D-glucoside hydrolase. Methods Enzymol 1997b; 280: 58-65.

61. European Commission, Health and Consumer Protection, Directorate-General. Directive 90/496/EEC on nutrition labelling for foodstuffs: discussion paper on revision of technical issues. http://ec.europa.eu/food/food/labellingnutrition/nutrition label/discussion_paper_rev_tech_issues.pdf [cited May 2006].

62. Bender MM, Rader JI, McClure FD. Guidance for industry: Food and Drug Administration Nutrition Labeling Manual - A Guide for Developing and Using Data Bases. United States Food and Drug Administration. http://www.fda.gov/Food/GuidanceComplianceRegulatoryInformation/GuidanceDocuments/ FoodLabelingNutrition/ucm063113.htm [cited 3 December 2007].

\footnotetext{
*Dr. Jesse F. Gregory

Professor of Food Science and Human Nutrition

PO Box 110370

University of Florida

Gainesville

FL 326 | |-0270, USA

Tel: +(0II) | 352392 |99| ext. 225

Email: jfgy@ufl.edu
} 